\title{
Cross-borehole resistivity tomography of Arctic and Antarctic sea ice
}

\author{
Keleigh JONES, ${ }^{1}$ Malcolm INGHAM, ${ }^{1}$ Daniel PRINGLE, ${ }^{2}$ Hajo EICKEN ${ }^{2}$ \\ ${ }^{1}$ School of Chemical and Physical Sciences, Victoria University of Wellington, PO Box 600, Wellington 6140, \\ New Zealand \\ E-mail: joneskele@myvuw.ac.nz \\ ${ }^{2}$ Geophysical Institute, University of Alaska Fairbanks, PO Box 757320, Fairbanks, AK 99775-7320, USA
}

\begin{abstract}
As an inhomogeneous mixture of pure ice, brine, air and solid salts the physical properties of sea ice depend on its highly temperature-dependent microstructure. Understanding the microstructure and the way it responds to variations in temperature and salinity is crucial in developing an improved understanding of the interaction between sea ice and the environment. However, measurements monitoring the internal structure of sea ice are difficult to obtain without disturbing its natural state. We have recently developed an application of cross-borehole d.c. resistivity tomography to make in situ measurements that resolve the anisotropic resistivity structure of first-year sea ice. We present results from measurements made in 2008 off Barrow, Alaska, and in 2009 off Ross Island, Antarctica. The sea ice in these two regions forms in different environments: at Barrow, relatively quiescent conditions typically lead to a predominance of columnar ice, while more turbulent conditions and underwater ice formation in McMurdo Sound tend to produce a larger component of frazil or platelet ice. Interpretation of the resistivity measurements carried out in association with temperature and salinity data collected simultaneously allows both observation of the temporal evolution of the ice structure and, in the case of the Antarctic measurements, the identification of different ice types.
\end{abstract}

\section{INTRODUCTION}

The bulk properties of sea ice are sensitive to the geometry and connectivity of the brine inclusions found within the solid ice matrix. These range in size from very small pores to large connected networks, depending upon temperature and salinity. An understanding of the manner in which the internal microstructure of sea ice changes in response to changes in temperature and salinity is therefore crucial for a fuller understanding of the properties of the sea-ice cover.

The existence and connectivity of brine channels in the ice affects the transport of heat through the ice (Perovich, 1998; Weeks, 1998; for example, Pringle and others (2007) have suggested that internal brine motion within the ice may contribute several percent to the overall heat flux. Brine inclusions also control the manner in which the ice interacts with electromagnetic radiation (Hallikainen and Winebrenner, 1992; Cherkaeva and Golden, 1998), while actual fluid permeability through pore spaces is important for nutrient transport through the ice (Fritsen and others, 1994).

Theoretical calculations of the physical properties of sea ice have generally been based on effective medium theories (e.g. Tinga and Voss, 1973) and, more recently, on percolation theory (Golden and others, 1998, 2006). Nevertheless there are few field measurements of the temporal variation in the physical properties of sea ice with which such theoretical calculations can be compared.

The internal structure of sea ice ought to be able to be studied using any transport property to which the brine and ice components contribute differently, for example nuclear magnetic resonance techniques (Callaghan and others, 1998, 1999; Eicken and others, 2000; Mercier and others, 2005), bail-test and fluorescent tracer methods (Freitag and Eicken, 2003), X-ray computed tomography (Kawamura, 1988; Pringle and others, 2009b) and impedance measurements (Pringle and others, 2009a; Notz and Worster, 2010).
However, such methods inevitably disturb the ice from its natural state and there are severe difficulties both in making accurate measurements and in interpretation.

Since there is a large contrast between the resistivity of the brine and solid ice components, measurements of electrical resistivity should yield information on sea-ice microstructure and, through this indirect probing, on other key ice properties. Direct current (d.c.) geoelectric soundings, widely used in shallow geophysical studies, are generally made using surface electrodes and therefore do not disturb the natural structure of the subsurface. However, due to the preferential vertical alignment of brine inclusions, the bulk resistivity of sea ice is anisotropic, with the vertical resistivity $\left(\rho_{\mathrm{v}}\right)$ being lower than horizontal resistivity $\left(\rho_{\mathrm{H}}\right)$. As a result, surface resistivity soundings are sensitive only to the geometric mean resistivity $\left(\rho_{\mathrm{m}}=\sqrt{\rho_{\mathrm{v}} \rho_{\mathrm{H}}}\right)$ of the medium (e.g. Bhattacharya and Patra, 1968). This has been noted clearly in previous resistivity measurements made on sea ice (e.g. Fujino and Suzuki, 1963; Thyssen and others, 1974; Timco, 1979; Buckley and others, 1986; Ingham and others, 2008) which have also shown that surface soundings underestimate the true thickness of the anisotropic sea-ice cover. This makes the interpretation of soundings, in terms of variation of resistivity with depth, problematic.

Recently Ingham and others (2008) have demonstrated that the d.c. resistivity technique of cross-borehole tomography, which has been used relatively widely in hydrological studies (e.g. Slater and others, 2000; Turner and Acworth, 2004; Looms and others, 2010; Morrow and others, 2010), can also be used to measure the horizontal component $\left(\rho_{\mathrm{H}}\right)$ of the anisotropic resistivity structure of sea ice. The technique involves making resistivity measurements using vertical strings of electrodes that have been frozen into boreholes in the ice while it is relatively thin and subsequently have been further embedded as the ice has 
thickened. Although freezing of electrode strings disturbs the ice structure immediately adjacent to the boreholes, the bulk of ice between the boreholes is undisturbed. In order to measure $\rho_{\mathrm{H}}$ it is necessary to use two boreholes, each containing one current and one potential electrode. In such measurements the current passes through the undisturbed ice between boreholes and therefore largely samples ice that is in its natural state.

Jones and others (in press) show that using four boreholes, each containing an electrode at approximately the same depth, allows estimates to be made of the geometric mean resistivity $\left(\rho_{\mathrm{m}}\right)$ of an infinite anisotropic medium. However, as sea ice is not an infinite medium but is bounded above by air and below by sea water, the geometric mean resistivity is underestimated, especially for thin and highly anisotropic ice. However, Jones and others (in press) show that for a known ice thickness and retrieved estimate of the mean resistivity, forward modelling of the anisotropic resistivity structure allows an estimate to be made not only of the true value of $\rho_{\mathrm{m}}$, but also of the degree of anisotropy $\left(\lambda=\sqrt{\rho_{\mathrm{v}} / \rho_{\mathrm{H}}}\right)$. From the values of the geometric mean and horizontal resistivity, estimates of the vertical component of the bulk resistivity, which cannot be measured directly, can also be obtained.

In this paper, we illustrate the use of the cross-borehole tomography technique to fully recover the anisotropic resistivity structure of sea ice, with examples from both the Arctic and the Antarctic. We demonstrate that the technique allows us not only to monitor changes in the microstructure of uniform ice of a single type, but also to distinguish between different ice types.

\section{FIELD MEASUREMENTS ON FIRST-YEAR SEA ICE}

Resistivity measurements were made in first-year sea ice at sites off the coast of Barrow, Alaska, during April-June 2008 and off Ross Island, Antarctica, in November 2009. Early in the growth of the ice, four electrode strings (as described in Ingham and others, 2008) were installed in boreholes drilled into the ice at the corners of a $1 \mathrm{~m}$ square. Using appropriate electrode arrangements (Jones and others, in press, measurements were made between each of the six borehole pairs. These allow a reasonable estimate of $\rho_{\mathrm{H}}$ in the volume contained by the boreholes to be derived by threedimensional (3-D) inversion. Similarly, measurements aimed at determining $\rho_{\mathrm{m}}$ were obtained using a four-electrode array with either each of the electrodes at approximately the same depth in separate boreholes, or three electrodes at the same depth in separate boreholes and a remote surface electrode. Inversion of these data yields an underestimate of the geometric mean resistivity from which the true value of $\rho_{\mathrm{m}}$ can be retrieved (Jones and others, in press).

At Barrow, the measurement site was on first-year landfast sea ice approximately $1 \mathrm{~km}$ off the coast. The University of Alaska Fairbanks (UAF) sea-ice mass-balance site was also operated at the same location $\left(71^{\circ} 21^{\prime} 56.45^{\prime \prime} \mathrm{N}\right.$, $\left.156^{\circ} 32^{\prime} 39.01^{\prime \prime} \mathrm{W}\right)$, recording snow and ice thickness, sea level, relative humidity and air, ice and water temperatures (Druckenmiller and others, 2009). Standard measurements were also carried out on sea-ice cores in order to determine salinity profiles.

In McMurdo Sound, the measurement site was also on first-year sea ice, formed as an extension of the Ross Ice Shelf, approximately $10 \mathrm{~km}$ from the coast of Ross Island. A mass-balance site was operated at the same site $\left(77^{\circ} 46^{\prime} 33.12^{\prime \prime} \mathrm{S}, 166^{\circ} 18^{\prime} 46.26^{\prime \prime} \mathrm{E}\right)$ as part of Antarctic New Zealand science event K131, providing measurements of temperature, ice thickness and snow depth and observations of platelet ice. As at Barrow, salinity data were obtained from ice cores.

\section{INVERSION MODELS}

\section{Arctic, 2008}

Figure 1 displays vertical sections through the horizontal and geometric mean resistivity structures, obtained from 3-D inversions of some of the 2008 Arctic data from Barrow. Also shown are corresponding plots of the variation with depth of both temperature and brine volume fraction, the former obtained from thermistor measurements and the latter calculated from the measured temperature and salinity profiles using the standard relationships given by Cox and Weeks (1983). During the inversion process the presence of sea water beneath the ice was incorporated using an initial model where, below the depth of the measured ice thickness, the resistivity was set to approximate that of sea water $(0.4 \Omega \mathrm{m})$. The degree to which resistivity values in this 'fixed' region could change was controlled during the inversions. Over the measurement period the ice thickness, measured by the UAF mass-balance site, increased from $1.28 \mathrm{~m}$ to $1.38 \mathrm{~m}$ before decreasing, during significant melt, to $0.83 \mathrm{~m}$ at the time of the last measurement.

The inversion modelling technique used does not necessarily produce unique models, so, as with all geophysical modelling, one must attempt to obtain physically realistic results and apply constraints from known parameters (e.g. ice thickness). The absolute misfit of the models shown in Figure 1 to the observed data varies from $2 \%$ to $15 \%$. However, in all cases most of the misfit occurs near the ice-water interface where steep gradients in resistivity occur which are difficult to model accurately with a numerical grid of finite size. Furthermore, assumptions made in the theory (Ingham and others, 2008; Jones and others, in press) mean it is worth considering the magnitude of resistivity changes that are likely to occur as the microstructure of sea ice changes due to expansion and connection of brine inclusions. A simple means of doing this is to consider the Hashin-Shtrikman limits (Hashin and Shtrikman, 1962) for the resistivity of a two-phase mixture comprising isolated spheres of resistivity, $\rho_{2}$, surrounded by a connected matrix of resistivity, $\rho_{1}$. For mixtures representative of sea ice it can be shown that for brine volume fractions of greater than about $0.5 \%$, the decrease in bulk resistivity between situations when brine pores are isolated ( $\rho_{2}$ is equal to the resistivity of brine, $\sim 0.330791 \Omega \mathrm{m}$ ) and when a connected network of brine exists ( $\rho_{1}$ is equal to the resistivity of brine) is 1-3 orders of magnitude. Given this order of magnitude for the likely changes in resistivity, it is clear that in terms of tracking the changes that occur in the microstructure of sea ice it is critical to be able to resolve between resistivity values of, for example, 20 and $200 \Omega \mathrm{m}$; however, it is not necessary to be able to discriminate accurately between values of, say, 200 and $400 \Omega \mathrm{m}$.

Although the geometric mean obtained from the inversions is an underestimation of the true value of $\rho_{\mathrm{m}}$, the degree of anisotropy, $\lambda$, can be retrieved using the underestimate of $\rho_{\mathrm{m}}$ and the value of $\rho_{\mathrm{H}}$ obtained from the inversion models 

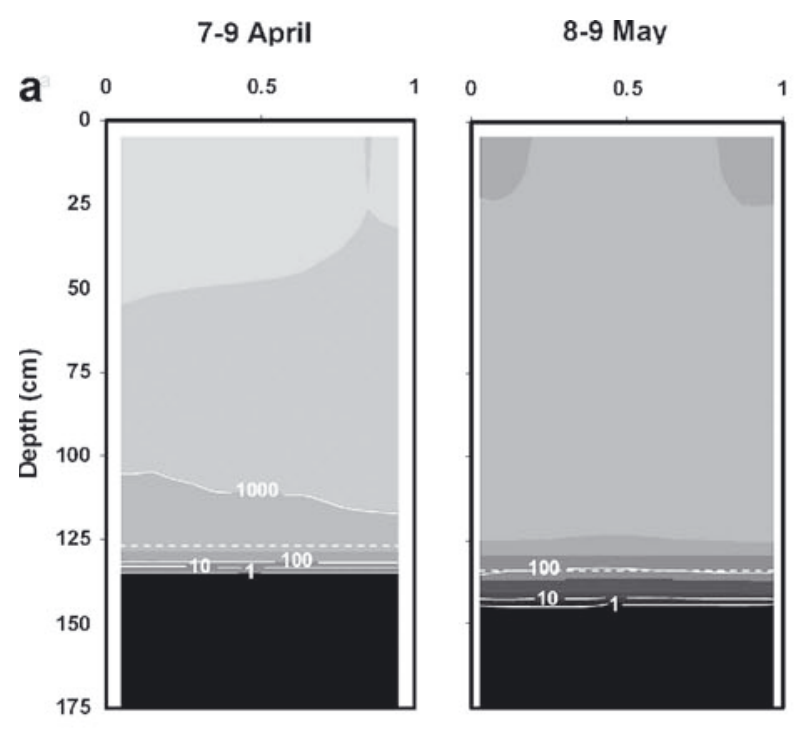

28-29 May
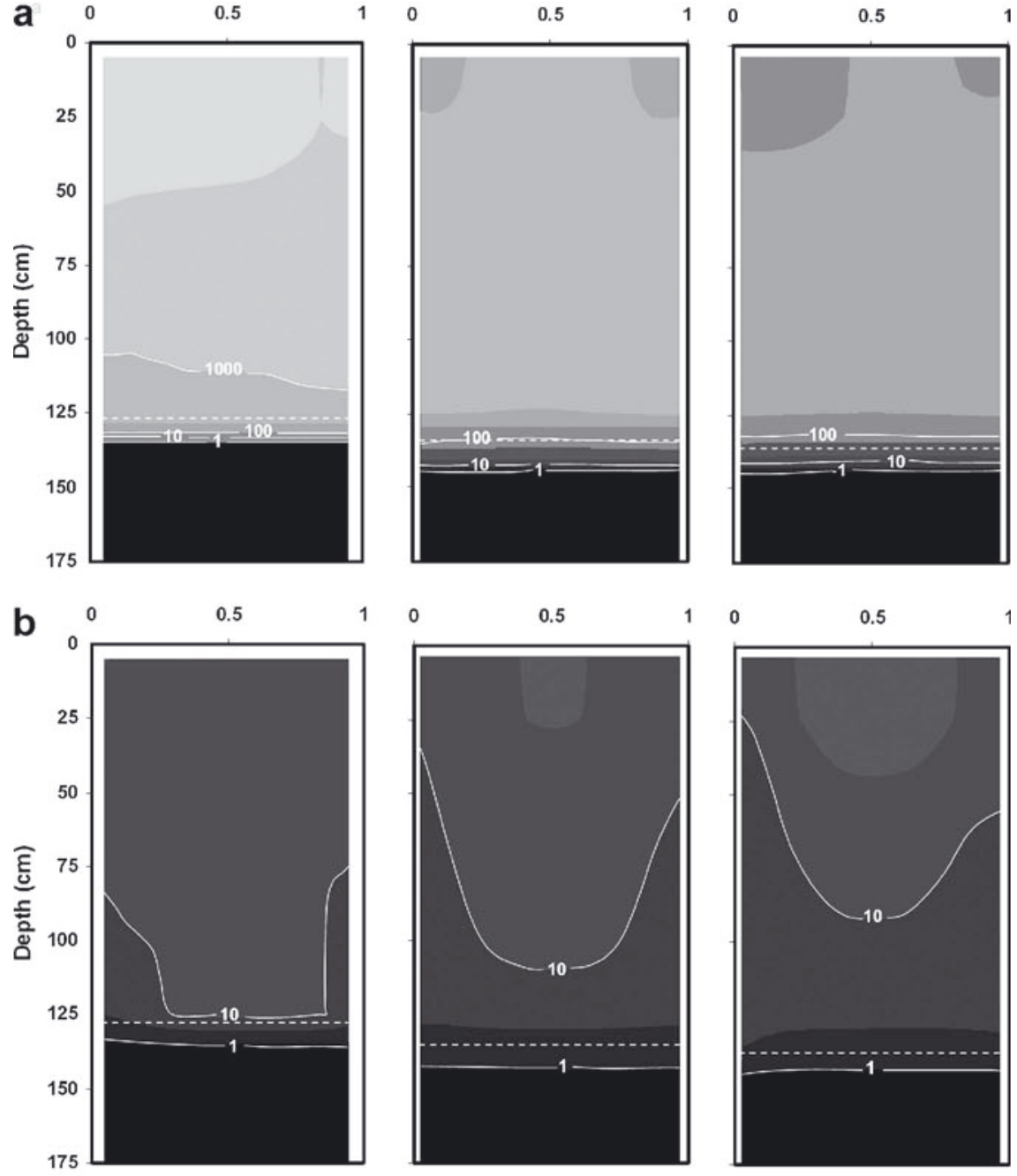

C
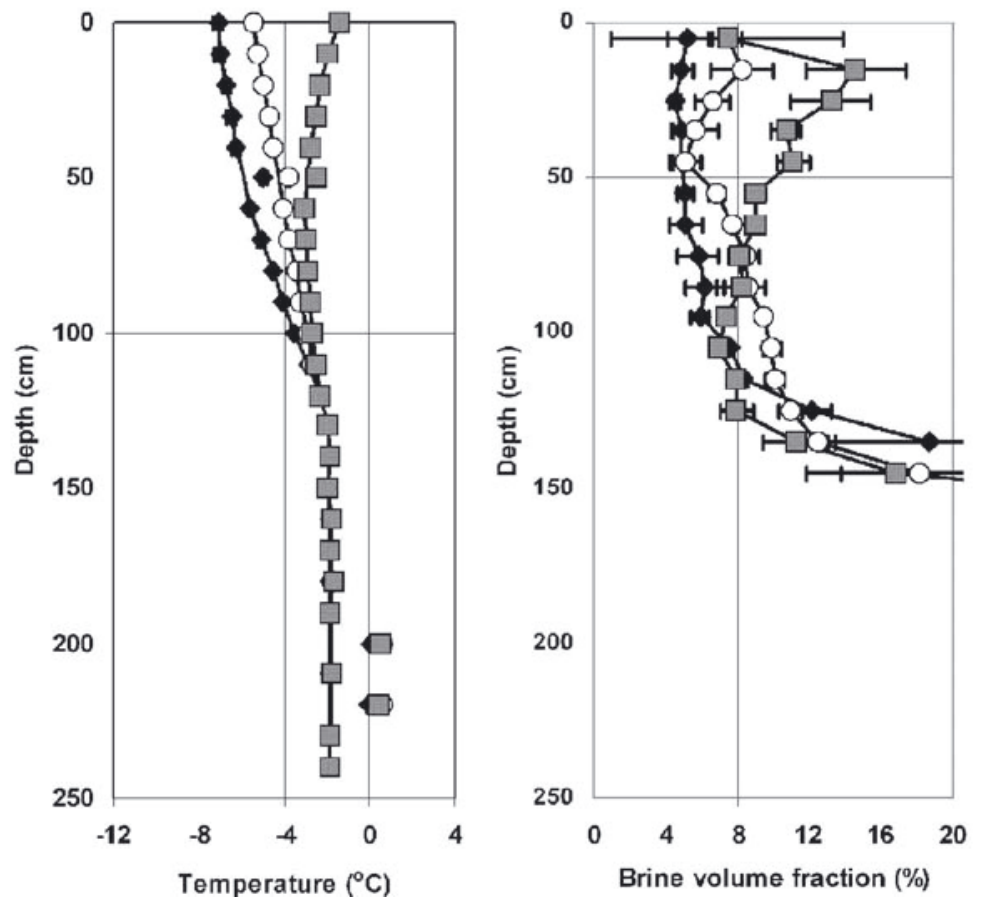

Fig. 1. (a) Vertical sections of the horizontal component of resistivity obtained from 3-D inversion of three datasets of the 2008 Arctic measurements. Contour values are in $\Omega \mathrm{m}$, and the ice-water interface is indicated by the dashed line. Horizontal distance is in $\mathrm{m}$. (b) As for (a) but for the geometric mean resistivity. (c) Vertical temperature and brine volume fraction profiles for the dates corresponding to the resistivity models in (a) and (b). The temperature profiles are obtained from thermistor measurements, and the brine volume fractions are calculated from the measured temperature and salinity profiles using the standard relationships given by Cox and Weeks (1983). Black diamonds: 7-9 April; white circles: 8-9 May; grey squares: 28-29 May. 


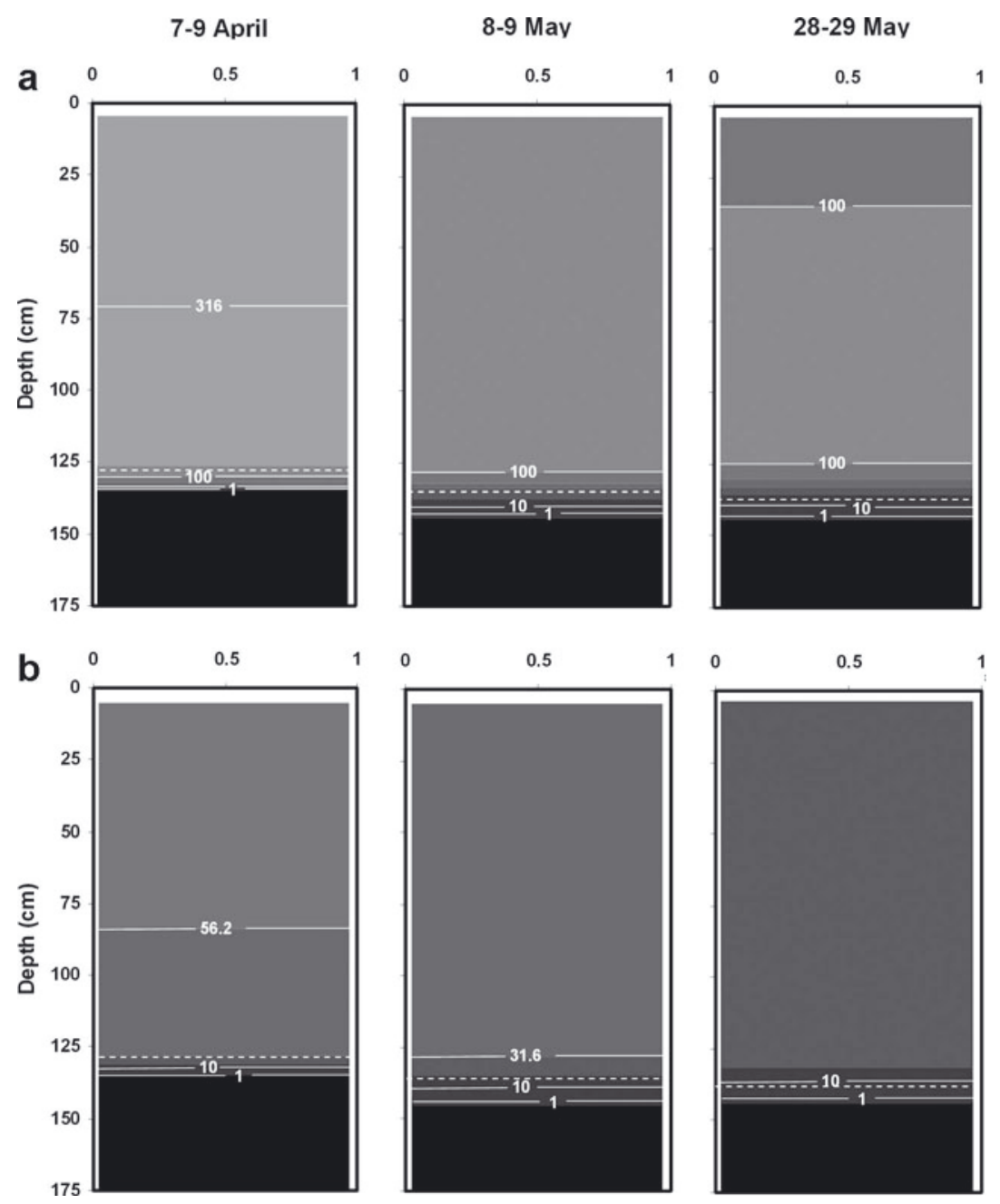

Fig. 2. (a) Vertical sections displaying the estimate of the true geometric mean resistivity for the datasets displayed in Figure 1 b. Contour values are in $\Omega \mathrm{m}$, and the ice-water interface is indicated by the dashed line. Horizontal distance is in $\mathrm{m}$. (b) As for (a) but for the vertical component of resistivity (calculated from $\rho_{\mathrm{H}}$ and $\rho_{\mathrm{m}}$ ).

(Jones and others, in press). The retrieved $\lambda$ can then be used with the model values of $\rho_{\mathrm{m}}$ to determine not only the true values of $\rho_{\mathrm{m}}$, but also to derive the vertical component of the bulk resistivity $\left(\rho_{\mathrm{v}}\right)$. Figure 2 shows the results of these derivations, displaying the true values of the geometric mean resistivity and the vertical resistivity structures.

From Figure 1 it can be seen that the horizontal component of the bulk resistivity displays high values of $300-1800 \Omega \mathrm{m}$. These values are within the range 200$5000 \Omega \mathrm{m}$ recorded by Ingham and others (2008) using cross-borehole measurements. Thyssen and others (1974) and Timco (1979) commented on measurements made using an array of electrodes located vertically on the wall of an ice pit to obtain the horizontal resistivity of sea ice. They quoted values of $221 \Omega \mathrm{m}$ and $129-298 \Omega \mathrm{m}$, respectively. Although these are rather low compared with our results from early spring, they are not dissimilar to those observed in mid- to late May after the ice has warmed. The general decrease observed in our resistivities as the temperature of the ice increases from early April to late May is likely to indicate a gradual change in the microstructure of the ice as connectivity of the brine pockets increases horizontally. Increased connectivity between brine pockets is also supported by an increase in brine volume fraction. Initially the resistivity structure gives high surface values which decrease gradually with depth until a sharp drop at the icesea interface. However, further into the melt season a region of low resistivity appears at the surface of the ice. This was also observed by Ingham and others (2008). The low resistivities combined with high surface temperatures and brine volume fractions suggest that surface warming has led to an increase in connectivity of the brine inclusions at shallow depth. By the time of the last measurement set, in early June, there had been significant surface melt and the top two to three electrodes were protruding from the ice surface. The resistivity profile displayed high surface resistivities, probably due to flushing of brine by meltwater. In the bulk of the ice the horizontal resistivity has decreased to low values of $60-70 \Omega \mathrm{m}$, indicating a significant change in the connectivity of the brine inclusions in the horizontal direction.

The values of the inversions of the geometric mean resistivity data (Fig. 1) are of the order of $10-20 \Omega \mathrm{m}$. However, the estimate of the true geometric mean resistivity retrieved through numerical modelling (Fig. 2) ranges from 100 to $350 \Omega \mathrm{m}$. These values are slightly higher than geometric mean resistivities obtained from previous studies which use surface soundings. For first-year sea ice near Pond Inlet, Northwest Territories, Canada, Thyssen and others (1974) obtained resistivities of the order of hundreds of $\Omega \mathrm{m}$, 
while Timco (1979) gave values of 76-176 $\Omega \mathrm{m}$. On Antarctic sea ice Buckley and others (1986) stated values of 50 $200 \Omega \mathrm{m}$. In our Barrow study, a number of surface resistivity soundings carried out adjacent to the location of the borehole measurements yielded geometric mean resistivities in the range $50-100 \Omega \mathrm{m}$, about a factor of 3 smaller than the geometric mean retrieved from the cross-borehole measurements. This lends some support to the suggestion that the cumulative effect of uncertainties and approximations in the retrieved $\rho_{\mathrm{m}}$ may mean that the values derived from the borehole measurements should be regarded as an estimate correct to within a factor of 3-4. Nevertheless, in the context of mapping the variation of resistivity structure with time over a series of measurement sequences, such an unresolved ambiguity may not necessarily be crucial.

The calculated vertical resistivity structure (Fig. 2) gives low resistivities of the order of 20-70 $\Omega \mathrm{m}$. This suggests a significant degree of vertical connection between brine inclusions even at the start of the measurement period when the ice was relatively cool. From ice-pit measurements Timco (1979) obtained values of 45-105 $\Omega \mathrm{m}$ for the vertical resistivity of first-year sea ice, comparable to the results presented here. Through the measurement period both the vertical and geometric mean resistivities decrease, but not as significantly as does the horizontal resistivity. In particular, although there is a large drop in horizontal resistivity observed between late May and early June, in the bulk of the ice the vertical resistivity remains relatively constant over the same period, with a maximum difference of $8 \Omega \mathrm{m}$ between the two measurement sets.

The bulk resistivity of the ice depends upon brine resistivity, brine volume fraction $\left(\phi_{\mathrm{b}}\right)$ and the structure of the brine inclusions. Defining formation factor as bulk resistivity over brine resistivity $\left(\rho / \rho_{\mathrm{b}}\right)$ combines these variables, so that a plot of formation factor vs brine volume fraction should give an indication of any changes that occur in the ice microstructure. Brine resistivity can be found using the measured temperature data and the expression given by Morey and others (1984). Figure 3 displays plots of $\left(\rho / \rho_{\mathrm{b}}\right)$ vs $\phi_{\mathrm{b}}$ for each of the horizontal, geometric mean and vertical components of the bulk resistivity, combining data from the three measurement dates shown in Figures 1 and 2. It can be seen in Figure 3 that there is a decrease in formation factor $\left(\rho / \rho_{\mathrm{b}}\right)$ and an increase in brine volume fraction $\left(\phi_{\mathrm{b}}\right)$ as the temperatures increased over the three measurement dates. Furthermore, the formation factor and hence the resistivity drops significantly between brine volume fractions of about $5-8 \%$. This large drop in resistivity for low brine volume fractions possibly corresponds to a percolation transition, marking a significant change in the microstructure of the ice. Above this transition (i.e. at higher brine volume fractions) the physical properties of the ice become dominated by those of the brine component. A similar result for vertical resistivity to that shown in Figure 3c has been found by K.M. Golden (personal communication, 2009) based on direct measurement of the vertical resistivity of Antarctic sea-ice cores.

The large change in formation factor and the occurrence of a percolation threshold have also been suggested by other studies. Golden and others (2007) suggested that macroscopic effects of vertical fluid permeability become significant when $\phi_{\mathrm{b}}$ is above about $5 \%$. Previous measurements of $\rho_{\mathrm{H}}$ (Ingham and others, 2008) have suggested that a significant drop in this resistivity occurs when $\phi_{\mathrm{b}}$ rises above about $8 \%$.
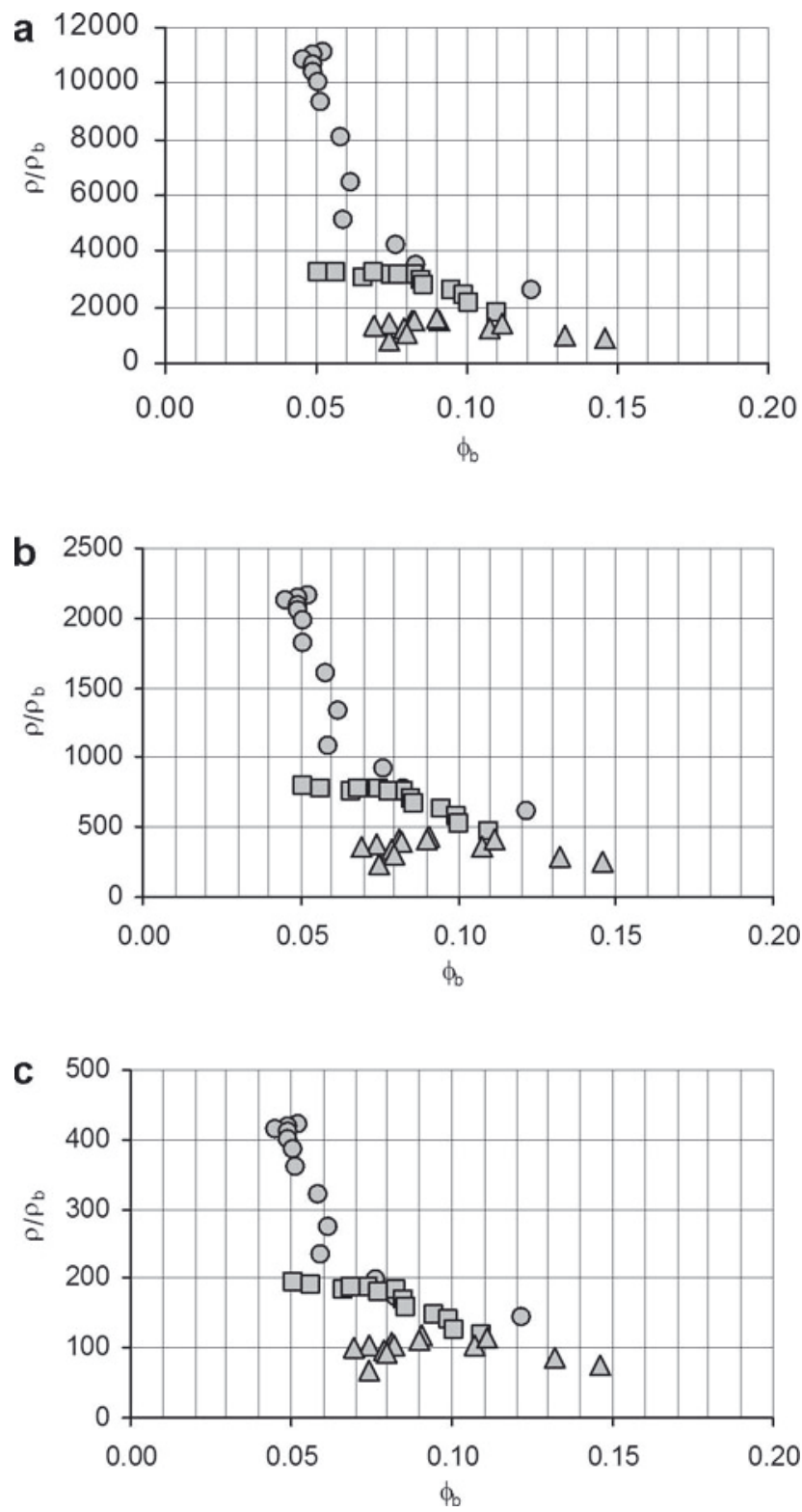

Fig. 3. Plots of formation factor $\left(\rho / \rho_{\mathrm{b}}\right)$ vs brine volume fraction $\left(\phi_{\mathrm{b}}\right)$ in \% for (a) horizontal resistivity, (b) geometric mean resistivity and (c) vertical resistivity. Circles: 7-9 April; squares: 8-9 May; triangles: 28-29 May.

\section{Antarctic, 2009}

Measurements in McMurdo Sound were made over a 2 week period relatively early in the spring warming. Over this period, although the ice warmed slightly, there was very little change in the measured resistivity structure. A vertical section of the horizontal component of the bulk resistivity, obtained from the 3-D inversion of the data measured on one particular date, is shown in Figure 4 and is representative of all the measured data. Also shown are temperature and brine volume fraction profiles. The ice thickness at the time of all the measurements was greater than the length of the electrode strings, so the ice-sea interface is not observed.

Figure 4 shows that the horizontal component of the bulk resistivity is of the order of hundreds to thousands of $\Omega \mathrm{m}$, similar to that observed in the early part of the sequence of the 2008 Arctic measurements. However, at depths below about $1 \mathrm{~m}$ there is a significant decrease in resistivity, which 

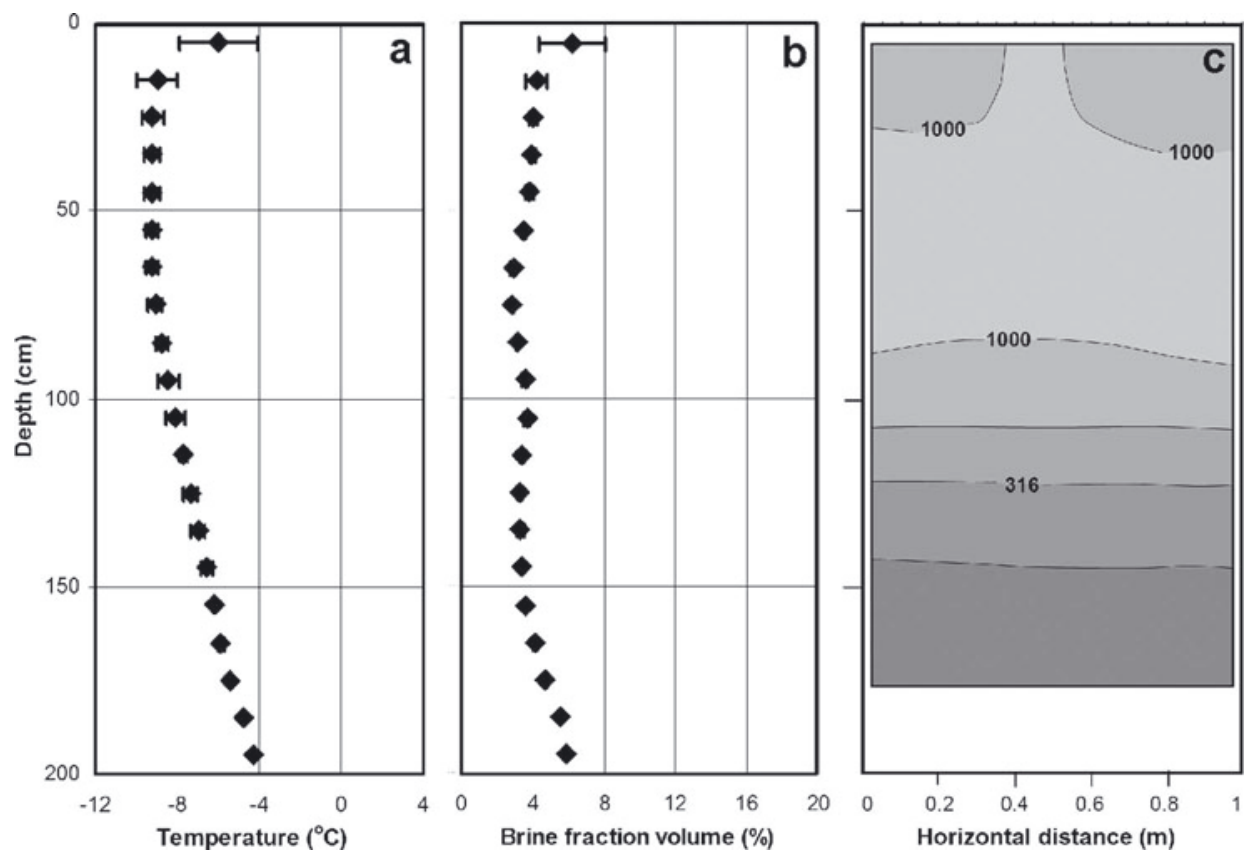

Fig. 4. Example of the 2009 Antarctic data. (a) Vertical temperature profile through the ice. (b) Brine volume fraction profile. (c) Vertical section showing the horizontal component of resistivity as obtained from 3-D inversion. Contour values are in $\Omega \mathrm{m}$. The ice-water interface at $\sim 2.4 \mathrm{~m}$ is not shown.

is not observed in the Arctic models. This does not correlate with any significant variation in either temperature or brine volume fraction. For example, between 1.0 and $1.5 \mathrm{~m}$ depth the calculated brine volume fraction is constant at about $3 \%$, and while there is a slight rise in temperature from about $-8^{\circ} \mathrm{C}$ at $1.0 \mathrm{~m}$ depth to $-6^{\circ} \mathrm{C}$ at $1.5 \mathrm{~m}$ depth, this is not regarded as large enough to result in significant temperaturecontrolled changes in microstructure. Such changes are predicted to occur (e.g. Golden and others, 1998; Golden, 2001, 2003) when the brine volume fraction reaches 5\% and the temperature reaches $-5^{\circ} \mathrm{C}$.

In the Antarctic, platelet ice can form beneath the ice cover in supercooled water, creating a tumbled entwined platelet matrix. This matrix can be incorporated into the ice cover as it grows downwards (Leonard and others, 2006; McGuiness and others, 2009; Dempsey and others, 2010). Such a region of included platelet ice could be expected to have a greater degree of horizontal connectivity between brine inclusions than that seen in columnar ice. The greater horizontal connectivity would be observed as a decrease in the horizontal component of resistivity. Thus, rather than representing a change in ice microstructure with temperature, we suggest that the observed resistivity decrease indicates a change in actual ice type from columnar ice, with little horizontal connectivity between brine inclusions at depths above $1 \mathrm{~m}$, to incorporated platelet ice beneath this depth. The base of any layer of incorporated platelet ice cannot be identified because at depths of about the limit of the resistivity section the brine volume fraction and temperature reach the critical values predicted by Golden (2003).

Preliminary analysis of the thin sections of ice cores, taken as part of Antarctic New Zealand science event K131, provides some support for this interpretation, with indications that platelet ice begins to appear at depths of 110$124 \mathrm{~cm}$ (personal communication from A. Gough and A. Mahoney, 2010). Additionally, over the course of the 2009
Antarctic winter, repeat conductivity-temperature-depth (CTD) casts through the ice identified significant periods when platelet ice was present in the water column beneath the growing ice cover.

\section{SUMMARY}

Sea ice is a complex medium with a microstructure that depends upon temperature, salinity and brine volume fraction. The structure is also affected significantly by the conditions under which the ice forms. For example, ice formed in generally quiescent conditions shows a significant columnar structure, while more active conditions lead to a more granular structure. As a result, significant differences in physical properties occur, which, given the importance of sea ice for climate, it is crucial to understand. Additionally, the preferential alignment of brine pores within the ice means that many of these properties are anisotropic.

The method of cross-borehole d.c. electrical resistivity tomography, more often associated with hydrological studies, permits in situ observation and monitoring of the microstructure of sea ice through direct measurement, retrieval or calculation of all the components $\left(\rho_{\mathrm{H}}, \rho_{\mathrm{m}}\right.$ and $\rho_{v}$ ) of the bulk resistivity structure. Resistivity structures, obtained from measurements made on first-year sea ice in the Arctic, show a change in the microstructure over a 3 month period as the ice warms during the spring melt. Over this period, all components of the bulk resistivity structure decreased, with the most significant decrease occurring in the horizontal component. This finding is interpreted as indicating an increase in the horizontal connectivity of the brine inclusions. Low vertical resistivity indicates that significant vertical connectivity of the brine inclusions exists even early in the season. Significant changes in the microstructure of the ice can be observed and suggest that a percolation transition occurs at a brine volume fraction of about $5-8 \%$. 
In contrast, Antarctic measurements display a significant decrease in resistivity with depth which is not observed in the Arctic data and does not appear to correlate with variations in temperature and brine volume fraction. We suggest that this decrease in resistivity corresponds to the inclusion of platelet ice into the sea-ice cover and this indicates that the cross-borehole method also may be used to differentiate between ice types.

\section{ACKNOWLEDGEMENTS}

K. Jones is supported by a Victoria University of Wellington Doctoral Scholarship/Assistantship. For the Arctic field measurements we acknowledge financial support through US National Science Foundation (NSF) Office of Polar Programs grants ARC-0620124 and 0934683 and logistic support provided by the Barrow Arctic Science Consortium. Antarctic field measurements were made as part of science event K131 coordinated by T. Haskell (Industrial Research Ltd, New Zealand). Logistics support was provided by Antarctic New Zealand. A. Gough and A. Mahoney of University of Otago, New Zealand, installed the electrode strings in McMurdo Sound. We thank the two reviewers and the scientific editor for comments on the manuscript.

\section{REFERENCES}

Bhattacharya, P.K. and H.P. Patra. 1968. Direct current geoelectric sounding: principles and interpretation. Amsterdam, etc., Elsevier.

Buckley, R.G., M.P. Staines and W.H. Robinson. 1986. In-situ measurements of the resistivity of Antarctic sea ice. Cold Reg. Sci. Technol., 12(3), 285-290.

Callaghan, P.T., C.D. Eccles, T.G. Haskell, P.J. Langhorne and J.D. Seymour. 1998. Earth's field NMR in Antarctica: a pulsed gradient spin echo NMR study of restricted diffusion in sea ice. J. Magn. Reson., 133(1), 148-154.

Callaghan, P.T., R. Dykstra, C.D. Eccles, T.G. Haskell and J.D. Seymour. 1999. A nuclear magnetic resonance study of Antarctic sea ice brine diffusivity. Cold Reg. Sci. Technol., 29(2), 153-171.

Cherkaeva, E. and K.M. Golden. 1998. Inverse bounds for microstructural parameters of composite media derived from complex permittivity measurements. Waves Random Media, 8(4), 437-450.

Cox, G.F.N. and W.F. Weeks. 1983. Equations for determining the gas and brine volumes in sea-ice samples. J. Glaciol., 29(102), 306-316.

Dempsey, D.E., P.J. Langhorne, N.J. Robinson, M.J.M. Williams, T.G. Haskell and R.D. Frew. 2010. Observation and modeling of platelet ice fabric in McMurdo Sound, Antarctica. J. Geophys. Res., 115(C1), C01007. (10.1029/2008JC005264.)

Druckenmiller, M.L., H. Eicken, M.A. Johnson, D.J. Pringle and C.C. Williams. 2009. Toward an integrated coastal sea-ice observatory: system components and a case study at Barrow, Alaska. Cold Reg. Sci. Technol., 56(2-3), 61-72.

Eicken, H., C. Bock, R. Wittig, H. Miller and H.O. Poertner. 2000. Nuclear magnetic resonance imaging of sea-ice pore fluids: methods and thermal evolution of pore microstructure. Cold Reg. Sci. Technol., 31(3), 207-225.

Freitag, J. and H. Eicken. 2003. Meltwater circulation and permeability of Arctic summer sea ice derived from hydrological field experiments. J. Glaciol., 49(166), 349-358.

Fritsen, C.H., V.I. Lytle, S.F. Ackley and C.W. Sullivan. 1994. Autumn bloom of Antarctic pack-ice algae. Science, 266(5186), 782-784.
Fujino, K. and Y. Suzuki. 1963. An attempt to estimate the thickness of sea ice by electric resistivity method. II. Low Temp. Sci., Ser. A 21, 151-157. [In Japanese with English summary.]

Golden, K.M. 2001. Brine percolation and the transport properties of sea ice. Ann. Glaciol., 33, 28-36.

Golden, K.M. 2003. Critical behavior of transport in sea ice. Physica B, 338, 274-283.

Golden, K.M., S.F. Ackley and V.I. Lytle. 1998. The percolation phase transition in sea ice. Science, 282(5397), 2238-2241.

Golden, K.M., A.L. Heaton, H. Eicken and V.I. Lytle. 2006. Void bounds for fluid transport in sea ice. Mech. Mater., 38(8-10), 801-817.

Golden, K.M., H. Eicken, A.L. Heaton, J. Miner, D.J. Pringle and J. Zhu. 2007. Thermal evolution of permeability and microstructure in sea ice. Geophys. Res. Lett., 34(16), L16501. (10.1029/2007GL030447.)

Hallikainen, M. and D. Winebrenner. 1992. The physical basis for sea ice remote sensing. In Carsey, F.D. and 7 others, eds. Microwave remote sensing of sea ice. Washington, DC, American Geophysical Union, 29-46.

Hashin, Z. and S. Shtrikman. 1962. A variational approach to the theory of the effective magnetic permeability of multiphase materials. J. Appl. Phys., 33, 3125-3131.

Ingham, M., D. Pringle and H. Eicken. 2008. Cross-borehole resistivity tomography of sea ice. Cold Reg. Sci. Technol., 52(3), 263-277.

Jones, K.A., M. Ingham, D.J. Pringle and H. Eicken. In press. Temporal variations in sea ice resistivity: resolving anisotropic microstructure through cross-borehole dc resistivity tomography. J. Geophys. Res. (10.1029/2009JC006049.)

Kawamura, T. 1988. Observations of the internal structure of sea ice by X-ray-computed tomography. J. Geophys. Res., 93(C3), $2342-2350$

Leonard, G.H., C.R. Purdie, P.J. Langhorne, T.G. Haskell, M.J.M. Williams and R.D. Frew. 2006. Observations of platelet ice growth and oceanographic conditions during the winter of 2003 in McMurdo Sound, Antarctica. J. Geophys. Res., 111(C4), C04012. (10.1029/2005JC002952.)

Looms, M.C., K.H. Jensen, A. Binley and L. Nielsen. 2010. Monitoring unsaturated flow and transport using cross-borehole geophysical methods. Vadose Zone J., 7(1), 227-237.

McGuiness, M.J., M.J.M. Williams, P.J. Langhorne, C. Purdie and J. Crook. 2009. Frazil deposition under growing sea ice. J. Geophys. Res., 114(C7), C07014. (10.1029/2007JC004414.)

Mercier, O.R., M.W. Hunter and P.T. Callaghan. 2005. Brine diffusion in first-year sea ice measured by Earth's field PGSENMR. Cold Reg. Sci. Technol., 42(2), 96-105.

Morey, R.M., A. Kovacs and G.F.N. Cox. 1984. Electromagnetic properties of sea ice. Cold Reg. Sci. Technol., 9(1), 53-75.

Morrow, F.J., M.R. Ingham and J.A. McConchie. 2010. Monitoring of tidal influences on the saline interface using resistivity traversing and cross-borehole resistivity tomography. J. Hydrol., 389(1-2), 69-77.

Notz, D. and M.G. Worster. 2010. In situ measurements of the evolution of young sea ice. J. Geophys. Res., 113(C3), 03001. (10.1029/2007JC004333.)

Perovich, D.K. 1998. The optical properties of sea ice. In Leppäranta, M., ed. Physics of ice-covered seas. New York, Springer-Verlag, 195-230.

Pringle, D.J., H. Eicken, H.J. Trodahl and L.G.E. Backstrom. 2007. Thermal conductivity of landfast Antarctic and Arctic sea ice. J. Geophys. Res., 112(C4), C04017. (10.1029/ 2006JC003641.)

Pringle, D., G. Dubuis and H. Eicken. 2009a. Impedance measurements of the complex dielectric permittivity of sea ice at 50 $\mathrm{MHz}$ : pore microstructure and potential for salinity monitoring. J. Glaciol., 55(189), 81-94.

Pringle, D.J., J.E. Miner, H. Eicken and K.M. Golden. 2009b. Porespace percolation in sea ice single crystals. J. Geophys. Res., 114(C12, C12017). (10.1029/2008JC005145.) 
Slater, L., A.M. Binley, W. Daily and R. Johnson. 2000. Cross-hole electrical imaging of a controlled saline tracer injection. J. Appl. Geophys., 44(2-3), 85-102.

Thyssen, F., H. Kohnen, M.V. Cowan and G.W. Timco. 1974. DC resistivity measurements on the sea ice near Point Inlet, N.W.T. (Baffin Island). Polarforschung, 44(2), 117-126.

Timco, G.W. 1979. An analysis of the in-situ resistivity of sea ice in terms of its microstructure. J. Glaciol., 22(88), 461-471.
Tinga, W.R. and W.A.G. Voss. 1973. Generalized approach to multiphase dielectric mixture theory. J. Appl. Phys., 44(9), 3897-3902.

Turner, I.L. and R.I. Acworth. 2004. Field measurements of beachface salinity structure using cross-borehole resistivity imaging. J. Coastal Res., 20(3), 753-760.

Weeks, W.F. 1998. Growth conditions and the structure and properties of sea ice. In Leppäranta, M., ed. Physics of icecovered seas. New York, Springer-Verlag, 25-104. 